\title{
Drinking functional coherent mineral water accompanies a strengthening of the very low frequency impact on heart rate variability, and mono and multifractal heart rhythm dynamics in healthy humans
}

\author{
Benny Johansson, Svetlana Sukhotskya
}

\author{
Akloma Bioscience AB, Medeon Science Park, 20512 Malmoe, Sweden
}

Corresponding author: Benny Johansson, PhD, Akloma Bioscience AB, Medeon Science Park, 20512 Malmoe, Sweden

Submission Date: May 16, 2016, Accepted Date: June 25, 2016, Publication Date: June 29, 2016

Study Design: A double blind, randomized pre-post cross-over clinical study.

\begin{abstract}
Background: Research on functional water has revealed its physiological functions and health-beneficial effects. Accordingly, this study explored the effects of drinking an average dietary volume of functional coherent mineral water (FCMW) on blood pressure, short-term cardiovascular variables, fractal heart rhythm dynamics, and salivary immunoglobulin A.
\end{abstract}

Methods: Fifteen healthy subjects were randomized in a pre-post crossover design, selected the morning after an overnight fast. Short-term electrocardiography (ECG) was measured before and after drinking either control mineral water (CMW) or FCMW on two separate occasions. Saliva was collected for four minutes before the start of each ECG measurement. Blood pressure was monitored in five-minute intervals for one hour.

Results: Drinking $100 \mathrm{ml}$ of FCMW temporarily increased arterial blood pressure at 20 to 25 minutes in the 60 minutes post-drinking. Drinking CMW led to a significant reduction in the heart rate, while all-time domain and Power Spectral Density parameters (PSD) were unaffected. Consumption of FCMW resulted in a highly significant difference and decrease in heart rate, and an increase in NN interbeat intervals, and in two of the PSD parameters. A large increase in total power had a significant increasing effect on 2-3-minute oscillations of the very low frequency (VLF) power, indicating a specific change in the heart's intrinsic cardiac rhythm that is fundamental to health and well-being. An extension in mono and multifractal scaling of heart rate dynamics defines a healthy function and non-local adaptability, indicating a higher capacity to respond to unpredictable stimuli and stresses, a function which improves autonomic stability. The effect on the heart rate and VLF power suggest an efficient cellular metabolism and a stabilizing effect on mucosal immunoglobulin A.

Conclusions: Functional coherent mineral water with an apparent non-local electromagnetic identity triggered in healthy subjects a sustainable sympathetic response in cardiac VLF 
power, which is considered an intrinsic health-promoting rhythm produced by the heart itself that accompanies adaptive mono and multifractal heart rhythm dynamics.

Keywords: functional water, VLF-power, heart rate variability, fractal scaling, health improvement

\section{BACKGROUND}

Based on the concept of homeostasis and physiological control, healthy systems are selfregulated to reduce variability, being accompanied by a constant "steady state" condition [1]. However, in contrast to the predictions of homeostasis for the outcome of a wide range of physiological systems such as the normal heart beat, heart rate, and blood pressure, etc., it has become abundantly clear that biological processes fluctuate in complex and nonlinear ways even under resting conditions [2]. These observations that physiological non-linear regulatory systems are operating far from equilibrium, in addition to the fact that maintaining constancy is not the purpose of physiological control, suggest the presence of fractal non-linear mechanisms and long-range, power-law correlations involved in physiological control and complex signalling networks to maintain physiological symmetry [2]. Water constitutes approximately $70 \%$ of total body mass and $99 \%$ of all molecules in humans [3], which aligns a non-linear quantum coherent regime of physiological water as an important factor in the emergence of self-organization and self-consistency of the living organism providing a mechanism for their non-locality $[4,5]$. Thus, functional water is defined as a form of water which promotes health, from which both treatment and physiological function have been scientifically demonstrated and shown reproducible, and where useful health-beneficial effects have been identified among natural and artificially treated waters [6].

\section{Heart rate variability and multi-functional scaling of heart rhythm}

The investigation of the heart's complex rhythms is defined as heart rate variability (HRV) measured by time domain and frequency domain (power spectral density) analysis. It is evaluated by means of short-term or 24-hour electrocardiograms (ECGs), non-invasive measures which indicate autonomic control associated with sympathetic and parasympathetic balance and cardiac activity [7]. The irregularity of the heartbeat of healthy systems as examined on a beat-to-beat basis under critical parameter conditions reveals a type of complex variability associated with long-range fractal correlations, along with distinct classes of non-linear interactions [2]. Multi-scaling, non-linear complexity appears to degrade in characteristic ways with aging and disease, reducing the adaptive capacity of the individual to self-regulate [2]. The presence of long-range correlations aligned with optimal HRV in cardiovascular fluctuations in health is associated with profound balance and adaptability or resilience [8-13] in both the sympathetic and parasympathetic control mechanisms that regulate beat-to-beat variability.

Consequently, physiological functional plasticity requires a broad range of alternatively integrated outputs, which defines the adaptability of a healthy function and the capacity to respond to unpredictable stimuli and responses. Comparable with HRV, fractal physiology expressed by long-range correlations in the heart beat as well as in gait dynamics, may be adaptive from two perspectives, $i$ ) long-range correlations serve as a self-organizing mechanism for highly complex fluctuations across a wide range of time scales, and ii) the absence of a characteristic scale inhibits the emergence of highly periodic, static and 
disruptive behaviours, which would greatly narrow functional responsiveness [2]. Therefore, self-regulative dynamics in health have implications connected with long-range oscillations and lowering of intrinsic entropy, giving rise to structural self-ordering and a state of physiological symmetry that implies that HRV follows allometric scaling laws [14, 15], which are considered as inherent characteristics of coherent dissipative structures $[16,17]$.

Among the four primary HRV frequency bands defined by the European Society of Cardiology and North American Society of Pacing and Electrophysiology - the highfrequency (HF), low-frequency (LF), very-low-frequency (VLF), and ultra-low-frequency (ULF) [18] - the VLF band with its power range between 0.003 and $0.04 \mathrm{~Hz}$, which relates to rhythms with periods that occur between 25 to 300 seconds, has historically been largely ignored even though it is most predictive of adverse outcomes [19-24]. Additionally, longterm regulatory mechanisms and autonomic nervous system (ANS) activity related to thermoregulation, the renin-angiotensin system, and other hormonal factors [25, 26], in recent work has shed new light on the primary mechanisms underlying the VLF rhythm [27-29]. The new findings imply that the VLF rhythm is generated by the stimulation of afferent sensory neurons in the heart, which in turn activates levels of the feedback and feed-forward loops in the heart's intrinsic cardiac nervous system, neurons in the extrinsic cardiac ganglia, and spinal column [30-31]. As a result, the VLF rhythm appears to be produced by the heart itself and may be an intrinsic rhythm that is fundamental to health and wellbeing (32). Subsequently, experimental evidence suggests that the VLF rhythm is generated by the heart intrinsically and that the amplitude and frequency of these oscillations are modulated by efferent sympathetic activity. Normal VLF power appears to indicate healthy function, whereas increases in resting VLF power and/or shifting of their frequency can reflect efferent sympathetic activity. The modulation of the VLF frequency due to physical activity, stress responses, and other factors that increase efferent sympathetic activation can cause it to cross over into the lower region of the LF band $[8,33]$.

\section{Coherent domains and non-local fields in water}

The dominant contribution of water molecules in molar terms and total mass in living systems makes water the apparent element which can give rise to a non-linear self-regulative quantum "super-coherent" state and to a nest of coherent domains (CDs) of water by inducing coherence among CDs forming a systemic axial coherence, such as the physiological water state of the human body [3,5]. There it exists at ambient temperature density fluctuations between a coherent low entropy and an incoherent distorted high entropy ordering water. The former is organized in spherical coherent domains [34, 35] of clustering water molecules tuning together in unison their quantum oscillations in phase with a self-trapped electromagnetic field (EMF) within the CDs [36]. The CDs can be activated to collect in their environment low grade energy, having a high entropy, and transform it, by exciting coherent vortices of almost free electrons, into high grade energy, with low entropy, that can release this energy outwards into useful work without thermal losses [4]. The unique value of such a super-coherent biological water spanning all the CDs throughout water is considered one of the most important characteristics of a healthy physiological state [37].

The function of water in the body is mainly classified according to $i$ ) the water molecule itself-where flowing water affects cellular function, and development and functions of organs [38, 39], and hydration and Brownian movement of water are fundamentally important for protein function; ii) atoms and molecules derived from water molecules; such 
as protons, hydrogen atoms (active hydrogen), hydrogen anions, hydrogen molecules, oxygen molecules, and reactive oxygen species (ROS) [40]; iii) molecules dissolved in water; such as mineral ions, mineral nanoparticles, organic and inorganic compounds, and gases [40]; $i v)$ the driving and regulatory role of water in governing the biochemical activity and bioenergetics, where CDs become electron donors providing electron transfer processes of aqueous systems [41]; and v) water as a liquid non-linear quantum medium; where liquid water molecules of CDs are assembled interconnected by time-dependent long-range radiative EMF, while short range static bonds, such as hydrogen bonds and electric dipole-dipole interactions, are the consequence of molecular condensation induced by these non-local fields [42, 43].

Functional water includes water used in biological contexts, in accordance with the definition of functional foods $[6,44]$. The functional quality of water restores self-regulative physiological conditions to maintain homeostasis $[45,46]$. Research on functional drinking water, especially reduced functional water, such as electrochemically reduced water [40] or natural reduced water [47] and functional coherent water [46] demonstrate profound physiological function associated with health. Reduced waters are defined to have preventive and positive effects on oxidative stress-related diseases, such as diabetes, cancer, arteriosclerosis, neurodegenerative diseases, and side effects of haemodialysis [6]. Functional water is activated water with or without a defined coherent state exhibiting specific functions. There are many activation methods without a defined ordered state of water, such as electrolysis, treatment with a magnetic field, light irradiation, ultrasonication, bubbling with gases, strong water flow and collision, treatment with specific types of minerals or rocks [6], and high pressure treatment [48]. The physical and chemical qualities of coherent water have been identified in bulk water when transformed by means of water splitting into a lasting excited high energy state of ordered water [49] and exposed to very mild ground state energy conditions, such as the influence of vortex agitation, freezing-thawing, evaporationcondensation, audio-sonication, capillary filtration, oscillating electromagnetic fields [50], water agitation with metal oxides [49], and influence of incident low entropy coherent sunlight after passing a Fibonacci optical lattice (FOL) (present study) [51].

Among functional waters, natural reductive mineral water (NRW) and electrolyzed water (EW) have been the most investigated. Potable NRW and EW are popular health-beneficial waters [6], which exhibit an alkaline $\mathrm{pH}$ [8-10], are highly reductive, have a negative oxidation-reduction potential, and perform reactive oxygen species (ROS) scavenging activity [49]. Clinical data suggested that EW improved oxygen stress-related diseases [52]. It was reported that in $45-\%$ of 320 patients with type 2 diabetes who drank 2 L natural Nordenau (Germany) mineral water per day, blood glucose and HBA1c levels were significantly decreased after 6 days. These levels were further decreased after long-term drinking [53]. In another open clinical study, 65 patients with diabetes and 50 patients with hyperlipidemia drank 2 L natural Hita Tenryousi (Japan) mineral water daily for two months. A significant decrease in blood sugar levels was observed in $89-\%$ of the patients. Blood triglyceride and total cholesterol levels with hyperlipidemia were significantly decreased in $92 \%$ of the patients [54]. Furthermore, in two double-blind random clinical trials, the first on 29 and the second on 100 type 2 diabetes patients who drank 1 L or 2 L Hita Tenryousi mineral water per day for 6 or 10 months respectively, urinary 8-OH dG (a marker of DNA oxidation) was significantly decreased in the first of the two studies [55]. Anti-metabolic syndrome effects were noted in the second study, such as a significant decrease in starved blood sugar levels, blood pressure, total cholesterol, LDL cholesterol, GOT, $\gamma$-GTP and 
triglyceride levels, arteriosclerosis index, uric acid levels, and a significant increase in leptin levels as well as improvement of constipation [56]. The clinical recommendation was that drinking up to 2 L NRW water per day was beneficial for health.

\section{Functional coherent water: a factor in physiological self-regulation}

Concerning functional coherent water, ingestion of a small volume, only one glass (100 ml), induced a bradycardial regulative response in healthy subjects, which was dependent on an increase in cardiac vagal activity, a rapid parasympathetic restorative response from the heart, and also significantly affected and caused a rise in salivary humoral immunity [46]. A concomitant increase in the power density in low frequency (LF) and high frequency (HF) power bands implied induction of a stabilizing resonance in autonomic regulation at frequencies in the range of $0.1 \mathrm{~Hz}$ induced by mid-range LF signals. Water intake had no effect on mean systolic and diastolic blood pressure. However, a distinct difference in blood pressure curve shape between the two waters was noted, with functional water characterized by a straight invariant horizontal line aligned along the entire time scale, and different from a slightly lower control blood pressure level, which suggested that functional water has a stabilizing effect on normal blood pressure. Additionally, a significantly lower variation in the standard deviation of systolic blood pressure might indicate fewer diurnal changes in blood pressure variability.

The autonomic adaptation to achieve homeostasis induced by functional liquid water was partly supported by previous findings, in which similar conditions created by gaseous coherent water in air transpired from indoor greenery led to an improvement in parasympathetic activation in healthy subjects during exposure to and discrimination of the stress load from an EMF from a visual display terminal [57]. The increase in parasympathetic activity was accompanied by an intensified spectral power band at frequencies in the range of $0.1 \mathrm{~Hz}$ in the presence of control or active (irrigated by vortex water) plants that led to a state of ANS stability. The EMF contributed to an increase in the amplitude of very low frequency (VLF) signals, stimulating physiologic arousal. However, exposure to natural gaseous plant cues generated a reduction in VLF power, which indicated the plant stimuli affect involuntary responses to EMF-induced distortion in PSD regulative signal processing, and that only active coherent plant cues induce qualities in air conditions equal to those of the control experiment before the EMF was applied (the percentage of VLF to total power was equal between the two experiments). Since both active and control plants have a more or less similar effect on cardiac and autonomic functioning, besides the striking normalization in VLF power by the active plant, an additional involuntary non-sensory self-adaptive state accompanying sensory intake or attention can be induced by natural or specifically by coherent VLF-quenching stimuli, which is consistent with the theory of restorative effects of natural objects [58-60].

Functional coherent water may be a significant factor in self-regulation and selfconsistency of homeostasis [46], based on the theory of dissipative systems' ability to selfregulate [16], and the assumption that VLF rhythms are involved in humoral and visceral regulation, which has been shown to be affected by depressing and upsetting thoughts, thereby serving as an indicator of a patient's achievement of a calm and peaceful internal state [61], optimizing coping ability due to inducing long-term hormonal response to external stressors [62], and the new recent findings, which imply that the VLF rhythm is intrinsically generated by the heart itself where normal VLF power indicates a healthy state [32]. The 
present study was designed to investigate whether a functional coherent water state in conformity with gaseous coherent water cues [57] may be a significant factor in accessing and maintaining the intrinsic VLF rhythm, specifically by examining the effects of consumption of an average dietary volume $(100 \mathrm{ml})$ of functional coherent mineral water on short-term (15 min) cardiovascular time and frequency PSD parameters, salivary immunoglobulin A (IgA), and on blood pressure in healthy subjects.

\section{METHODS}

Fifteen (15) healthy non-smoking subjects, eight females and seven males aged between 18 and 58 years (mean age 36.5 years), participated in the study. Subjects were normotensive, were not on medication, and were screened by means of their history and physical examination to exclude cardiovascular and neurological dysfunction (BMI was measured and notified). They were recruited from the employees of Scientific Park corporations. The study protocol followed the approved standard of the local ethics committee and the Helsinki declarations of 1983 and 1995. Written informed consent was obtained.

\section{Study design}

The subjects were studied using a double blind randomized pre-post crossover design in the morning after an overnight fast. Subjects were studied individually, one participant per day. Individuals receiving CMW during the first part of the study were given FCMW during the second part and vice versa. Controls were measured prior to drinking either test water. During the study, the subjects were seated in ordinary working position on an office chair in front of a table. The room was air-conditioned with a constant temperature around $25^{\circ} \mathrm{C}$.

\section{Assessment}

Electrocardiography (ECG) was performed after the participants had undergone an initial conditioning period of sitting at the table for 15 minutes and measured from the wrists via sensor electrodes connected to a Heart Rhythm Scanner (Biocom Technologies, US). Sensor pads marked "left" and "right" were placed on the respective wrist with the electrode disks facing downwards and firmly secured by means of elastic wristbands. Short-term measurements (15-minute sessions) of time domain and power density parameters (PSD) were performed and analyzed by a special computer software program, according to standard guidelines approved by the European and North American Societies of Cardiology (18). Time domain parameters were calculated from the heart rate and determined by R-R interbeat intervals. The time domain analysis recordings were mean heart rate (mean HRT), mean of the normal to normal heartbeats (mean $\mathrm{NN}$ ), standard deviation of the normal to normal heartbeats (5-minSDNN; the term SDNN will be used in the following text), the square root of the mean squared differences of successive N-N intervals (RMSSD), and an estimate of the parasympathetic activity of the heart. The PSD analysis recalculates the frequency content of time domain data into three distinct frequency segments. Each frequency range represents different segments of the autonomic nervous system and provides information about frequency domain terms as follows: Total power, which is an estimate of the total power of all $\mathrm{NN}$ intervals over the record length in the range of frequencies between 0-0.4 Hz; very low frequency power, involving sympathetic components less than or equal to $0.04 \mathrm{~Hz}$ (VLF); low frequency power, which measures both sympathetic and parasympathetic components equal to $0.04-0.15 \mathrm{~Hz}(\mathrm{LF})$; and high frequency power, which equals $0.15-0.4$ 
$\mathrm{Hz}$ (HF) and relates to parasympathetic or vagal activity. Normalized low- and highfrequency units (LF norm and HF norm) express the ratio between the frequency of interest and the difference between total power and VLF multiplied by 100. The ratio of LF to HF power quantifies the absolute LF to absolute HF power (LF/HF).

After connection of the ECG equipment, the subjects were allowed to rest for $15 \mathrm{~min}$. Saliva was collected for 4 min prior to the start of a 15-min HRV and PSD recording. Subjects were then asked to drink $100 \mathrm{ml}$ of control mineral or functional water (temperature $20-{ }^{\circ} \mathrm{C}$ ) in a relaxed manner. The bottom of the bottles with control and functional water was labelled with a numerical code, which was uncovered after completion of the experimental part of the study in order to identify the type of water. Control mineral or functional coherent water was randomly and double-blind assigned between subjects in the first part of the study. A second portion of control mineral or functional coherent water was administered approximately two weeks later. Those subjects given control water during the first part of the study received functional water on the second occasion and vice versa. After conditioning for $15 \mathrm{~min}$, another saliva sample was collected 4 min prior to the start of the final 15 min HRV and PSD recording.

Detrended fluctuation analysis (DFA) [2, 63] determines the monofractal scaling behavior of numeric, e.g. time series data, in the presence of possible trends without knowledge of their origin. The DFA algorithm permits the detection of intrinsic selfsimilarity embedded in a non-stationary time series. The root mean square fluctuation of the integrated and detrended time series $F(n)$ behaves as a power law function of $\mathrm{n}$ :

\section{$\left(F(n) \alpha n^{d}\right)$}

The integrated time series is divided into boxes of equal length $n$. For each box of length $n$, a least square line is fitted to the data. The calculation is repeated over all time scales (box sizes). The DFA exponent $(d)$ is defined as the slope of the regression line log $n$ vs. $F(n)$. A DFA exponent ranging from $0.5 \leq d \leq 1$ indicates long-range power law correlations, growing stronger towards 1.

Multifractal characterization of nonstationary time series is based on a generalization of the DFA method and is extended to temporal variation in scale invariant structures, which indicate a multifractal structure of time series data that is defined by a multifractal spectrum of power law exponents [63-65]. In order to quantify possible differences in singularity spectra stemming from different structural signals, it is possible to fit by the least square method the spectra to a quadratic function around the position of their graph maxima at $\alpha_{0}$, according to a multifractal spectrum $F(\alpha)$ :

$$
F(\alpha)=A\left(\alpha-\alpha_{0}\right)^{2}+B\left(\alpha-\alpha_{0}\right)+C
$$

Parameter $B$ indicates the asymmetry of the curve, which is zero for symmetric, positive for left-skewed, and negative for right-skewed shapes. A right-skewed spectrum denotes the relatively strong dominance of high fractal components, corresponding to rough fractal structures and a left-skewed spectrum indicates low smooth-looking fractal structures. To obtain an estimate of the width of the spectrum for the range of $\alpha$ where $F(\alpha)>0$, the fitted curves are extrapolated to zero. The width of the spectrum is defined:

$$
\Delta \alpha=\alpha_{\max }-\alpha_{\min } \text { with } F\left(\alpha_{\max }\right)=F\left(\alpha_{\min }\right)=0
$$


The higher the $\Delta \alpha$, the wider the range of fractal exponents in the signal and the richer the process in structure. Consequently, a wider opening of the graph indicates a non-uniform clustering structure of sequences. The difference in fractal dimensions of the maximum probability subset $\left(\alpha=\alpha_{\max }\right)$ and the minimum $\left(\alpha=\alpha_{\min }\right)$ is $\Delta f\left(\Delta f=f\left(\alpha_{\min }\right)-f\left(\alpha_{\max }\right)\right) . f\left(\alpha_{\min }\right)$ and $f\left(\alpha_{\max }\right)$ reflect the number of the subset of the maximum and minimum probability, respectively. Thus, $\Delta f<0$ represents that the chance of the interbeat intervals lying at the lowest site is more than that at the highest site and vice versa.

The monofractal DFA exponent and multifractal $(\Delta \alpha, B, \Delta f)$ parameters can be used as quantitative dynamical indexes of functional coherent water reflecting the extension in mono and multifractal scaling of heart rate dynamics, a feature of a healthy function [2]. The change in the DFA [63] and multifractal exponents [64-66] were calculated on mean N-N interbeat intervals from individual ECGs. Multifractal DFA was performed using a VBA script for Microsoft [67]. The frequency spectrum of VLF time series data was evaluated by Fourier analysis [68].

Secretory saliva was collected in accordance with regular methodology [69]. Approximately one millilitre of unstimulated whole saliva was collected over a four-minute period and stored frozen at $-20-{ }^{\circ} \mathrm{C}$ until assayed. The salivary immunoglobulin A (IgA) concentration was analyzed and quantified in duplicate using an accredited standard immunoassay [70].

Arterial blood pressure was obtained as the mean of triplicate consecutive measurements at five-minute intervals over a one-hour period using a blood pressure monitor (Omron RX, Matsuaka Co. Ltd, Japan). The first measurement was taken immediately before ingestion and the second 10 minutes after drinking, followed by repeated measurements every five minutes.

The mineral water was obtained directly from the producer (Aqua Terrena spring water, Sweden). Functional coherent qualities were obtained by incident daylight passing a transparent topographical FOL following the proportions of the golden section and Fibonacci series of numbers [51, 71, 72]. The two-dimensional geometrical grid imprinted on a polyethylene terephthalate (PET) substrate causes ordinary photons to self-organize spatially with fractal symmetry, lower entropy, and higher intensity at ordinary temperatures and pressures $[3,36,37]$. The structure design is based on the geometry of the concentric circle, wherein there must be a specific relationship in accordance with the proportions of Phi $[1,68]$ or Fibonacci's sequence of numbers between the outer diameter of the circle and the next circle, counting inwards towards the common centre of circles. The line widths constituting the geometrical matrix equally follow the proportion of Phi. The FOL works as a fractal spatial-wave "Fourier raster" and is capable of transforming the spatial dissonance of electromagnetic field oscillations into harmonic wave components. Incident visible or thermal light that transmits the raster activates the fractal field due to the interference of the fluxes transmitted through and reflected by the diffraction lattice of the geometry. The light fields transform into a nonlinear series of wave structures with fractal coherent ordering in the visible and infrared wavelength range of light spectrum. Quantum fluctuations are captured by incident photons, giving rise to an excited highly ordered quantum state, where quantum coherent photons are self-trapped and accumulated with extended CDs that prescribe a co-oscillation with water molecules in the liquid state. The coherent state of liquid water is a reorganized field-like ordered unification of bulk water molecules with non-local 
identity. Among the features of coherent water is that extended water clusters aggregating in an auto-stabilizing network can grow to macroscopic proportions $[57,50,73]$ and affect the condition of any external water in a liquid or gaseous state [74-76]. The functional coherent water was obtained by exposing the mineral water stored in a PET bottle to the topological FOL imprinted on the wall of the bottle at ordinary daylight and ambient conditions for $24 \mathrm{~h}$ before ingestion.

\section{STATISTICAL ANALYSIS}

The study design aimed to compare the variance in mean values evaluated from individual changes in HRV and PSD parameters, arterial blood pressure, and salivary IgA. Mono and multifractal scaling were evaluated from the difference of the mean values between groups. Statistical analysis was performed by means of the nonparametric two-tailed Wilcoxon signed-rank test for estimation of significance across each group. Linear regression was used to model the outcome of BMI on HRV and PSD measures. Data are presented as mean \pm S.D. and a probability level of $p<0.05$ was considered significant.

\section{RESULTS}

Drinking $100 \mathrm{ml}$ of functional coherent water did result in a small change and increase in arterial systolic blood pressure at 20 minutes $(\mathrm{p}=0.027)$ and 25 minutes $(\mathrm{p}=0.031)$ and in diastolic blood pressure at 20 minutes $(\mathrm{p}=0.038)$ post-drinking (Figure 1). Notably, the invariance between the two blood pressure curves was characterized as a straight horizontal line between 10 and 60 minutes. Mean baseline (between 10 and $60 \mathrm{~min}$ ) systolic (119.4 \pm $1.3 \mathrm{mmHg})$ and diastolic blood pressures $(75.8 \pm 0.9 \mathrm{mmHg})$ were slightly but significantly lower after ingestion of functional coherent water; systolic $121.5 \pm 0.9(p<0.001)$ and diastolic blood pressure $77.2 \pm 0.9 \mathrm{mmHg}(\mathrm{p}<0.001)$.

Consumption of $100 \mathrm{ml}$ control mineral water did affect two of the time domain ECG parameters, while ingestion of functional coherent mineral water affected two additional PSD parameters (Table 1). A significant fall in heart rate of controls $(p=0.016)$ and experimental subjects $(p=0.002)$ accompanied a concomitant increase in RR interval between heart beats of controls $(\mathrm{p}=0.021)$ and experimental subjects $(<0.001)$. A significant increase in total power $(\mathrm{p}=0.013)$ and the VLF power band $(\mathrm{p}=0.013)$ indicated that functional coherent mineral water may influence stimulation of afferent sensory neurons in the heart. The percentage of the ratio of the VLF to total power revealed a very clear shift from a VLFdiscriminated condition after the ingestion of control mineral water ( $46.9 \%$ to $38.6 \%$ ) to an opposed and stimulating self-regulative and self-sustainable response caused by functional coherent mineral water ( $45.6 \%$ to $50.5 \%$ ).

Fourier analysis of the mean value (all 15 participants) of the mean difference of VLF signals between control mineral water (CMW) minus control 1 (before intake of CMW) and functional coherent mineral water (FCMW) minus control 2 (before intake of FCMW) are shown in Figure 2. The main difference between the two curves is the dominant peak around $0.003 \mathrm{~Hz}$ after ingestion of functional mineral water (right curve). The distribution of low VLF signals 
A

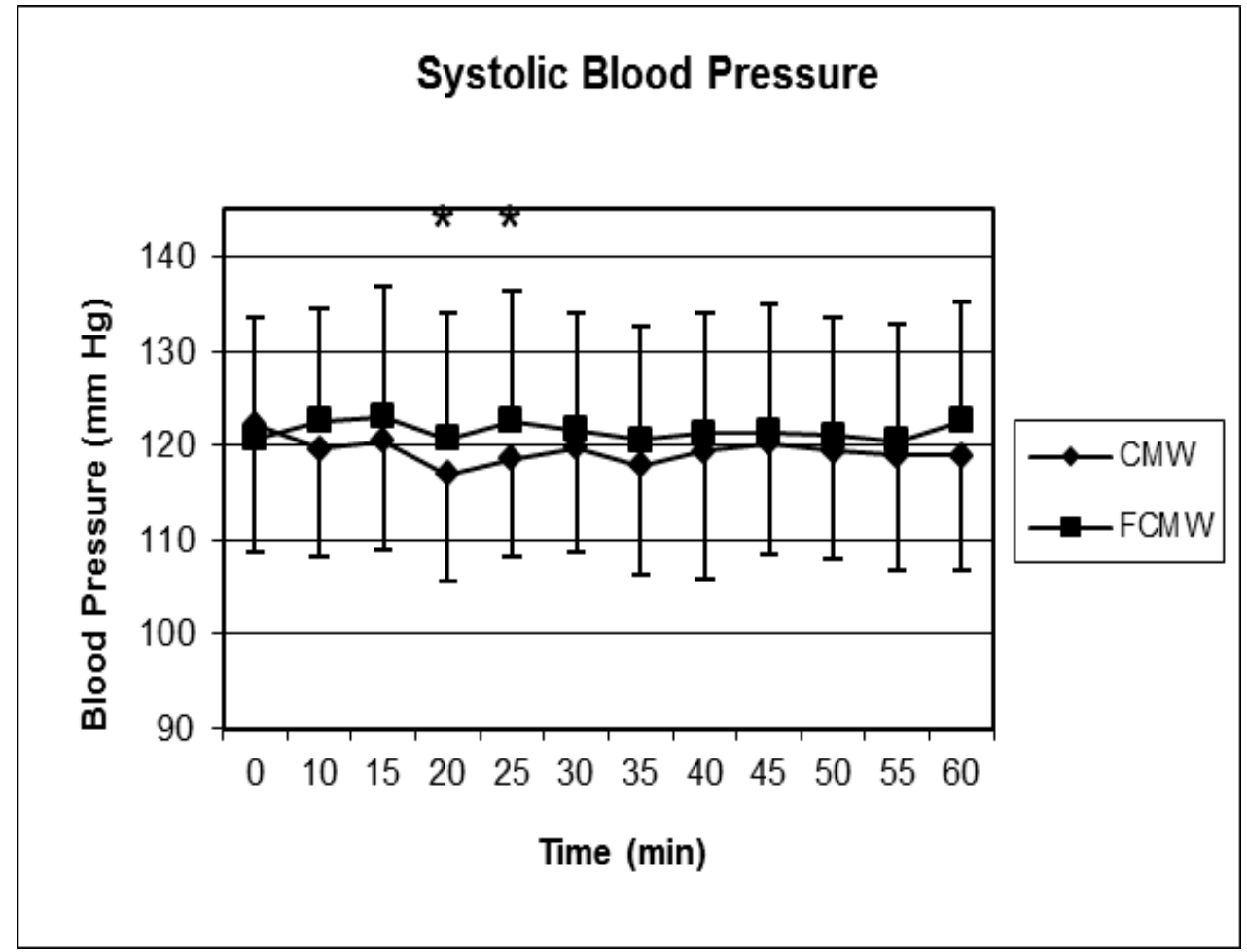

B

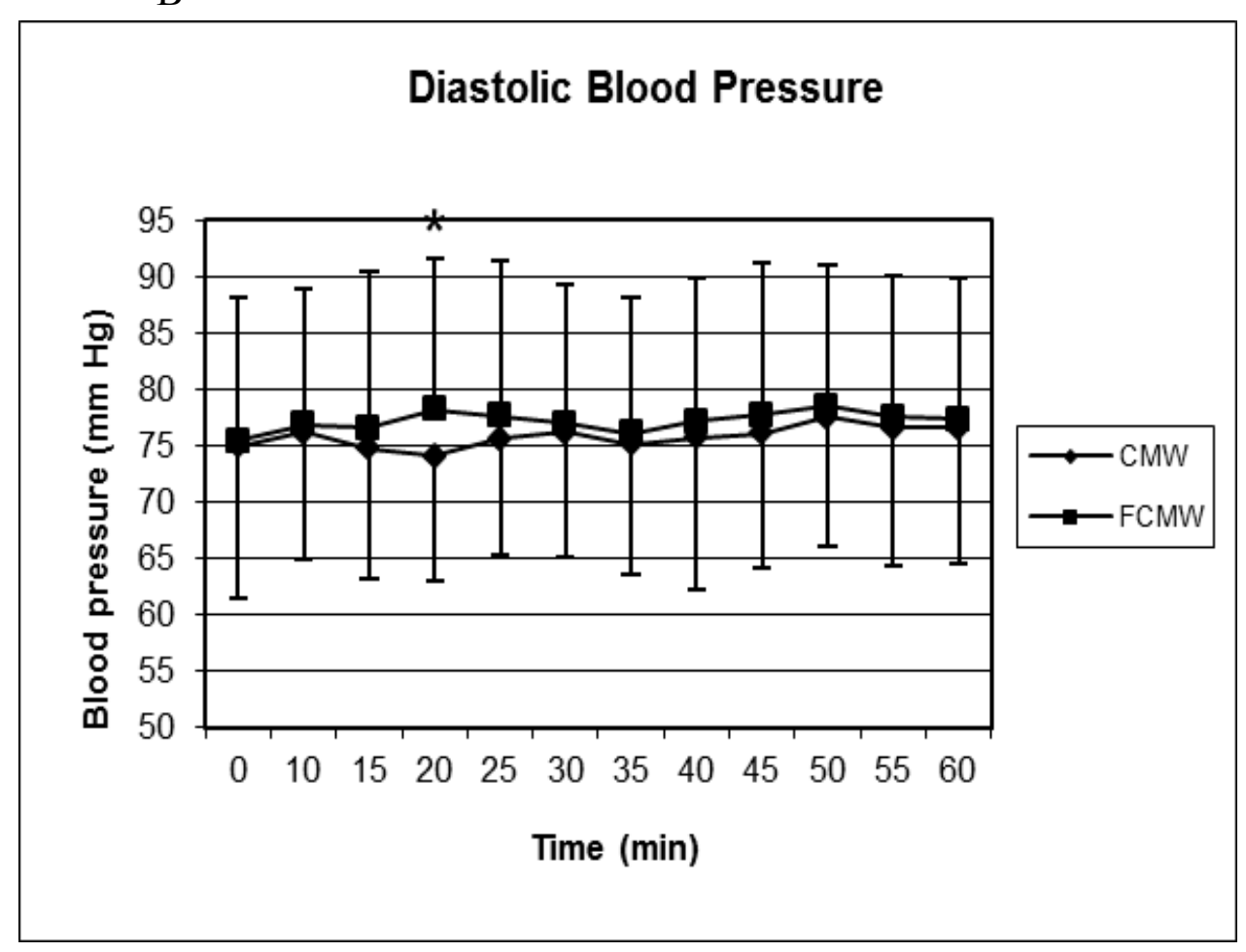

Figure 1. Systolic (A) and diastolic blood pressure (B) in healthy subjects following ingestion of control mineral water (CMW) or functional coherent mineral water (FCMW) $(* \mathrm{~A} 20 \min \mathrm{p}=0.027 ; 25 \min \mathrm{p}=0.031 ; * \mathrm{~B} 20 \min \mathrm{p}=0.038)$. 

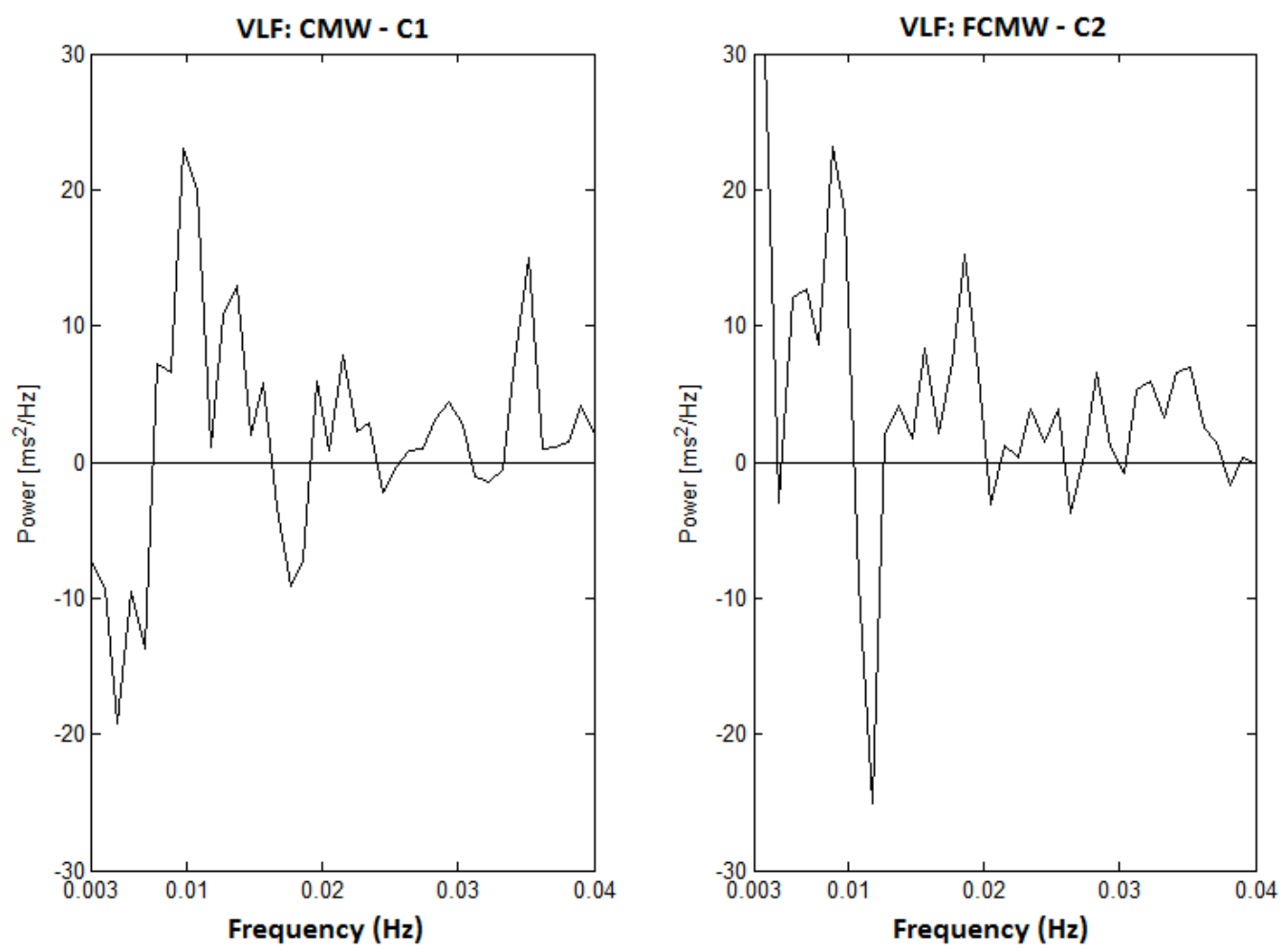

Figure 2. Fourier analysis of VLF signals from 15 min ECG measurements, following ingestion of control mineral water (CMW) (left curve) and functional coherent mineral water (FCMW)(right curve). The recording is the mean value (of all 15 participants) of the mean difference between CMW - Control 1 or FCMW - Control 2, respectively. The main difference between CMW and FCMW is the VLF peak around $0.003 \mathrm{~Hz}$ (right curve).

$(0.98-7.82 \mathrm{mHz})$ revealed (Figure 3$)$ that intake of control mineral water compared to control 1 values generated generally higher VLF amplitudes with the maximum peak at $1.95 \mathrm{mHz}(\lambda$ $=8.55 \mathrm{~min}$ ). Ingestion of functional coherent mineral water showed a shift towards stimulation of VLF signals with higher maximum frequencies at $5.58 \mathrm{mHz}(\lambda=2.99 \mathrm{~min})$ and $7.82 \mathrm{mHz}(\lambda=2.13 \mathrm{~min})$. Control 2 values showed a peak at $2.93 \mathrm{mHz}(\lambda=5.69 \mathrm{~min})$. The results indicate that drinking functional coherent mineral water stimulated a shift of VLF signals towards higher frequencies and increased coherent energy with a proposed biological impact, which could support the idea that control mineral and functional coherent mineral water act on cardiovascular regulation by means of activation of different signals within the VLF power band.

The mono and multifractal scaling relationship of the heart interbeat interval time series after ingestion of functional coherent mineral water (Table 1) demonstrated fractal scaling, with the mono scaling exponent DFA significantly increased $(p=0.044)$ compared to the control mineral water with a lowered DFA, which indicated that interbeat signals are characterized by high persistence or long-term memory. From the multifractal analysis, the multifractal scaling exponent $\Delta \alpha$ was significantly increased $(\mathrm{p}=0.028)$, which indicated a non-uniform clustering structure of the sequences. Relative to control mineral water, the higher $\Delta \alpha$ in functional coherent water shows that the variation of interbeat intervals is larger and that the multifractality is stronger. 


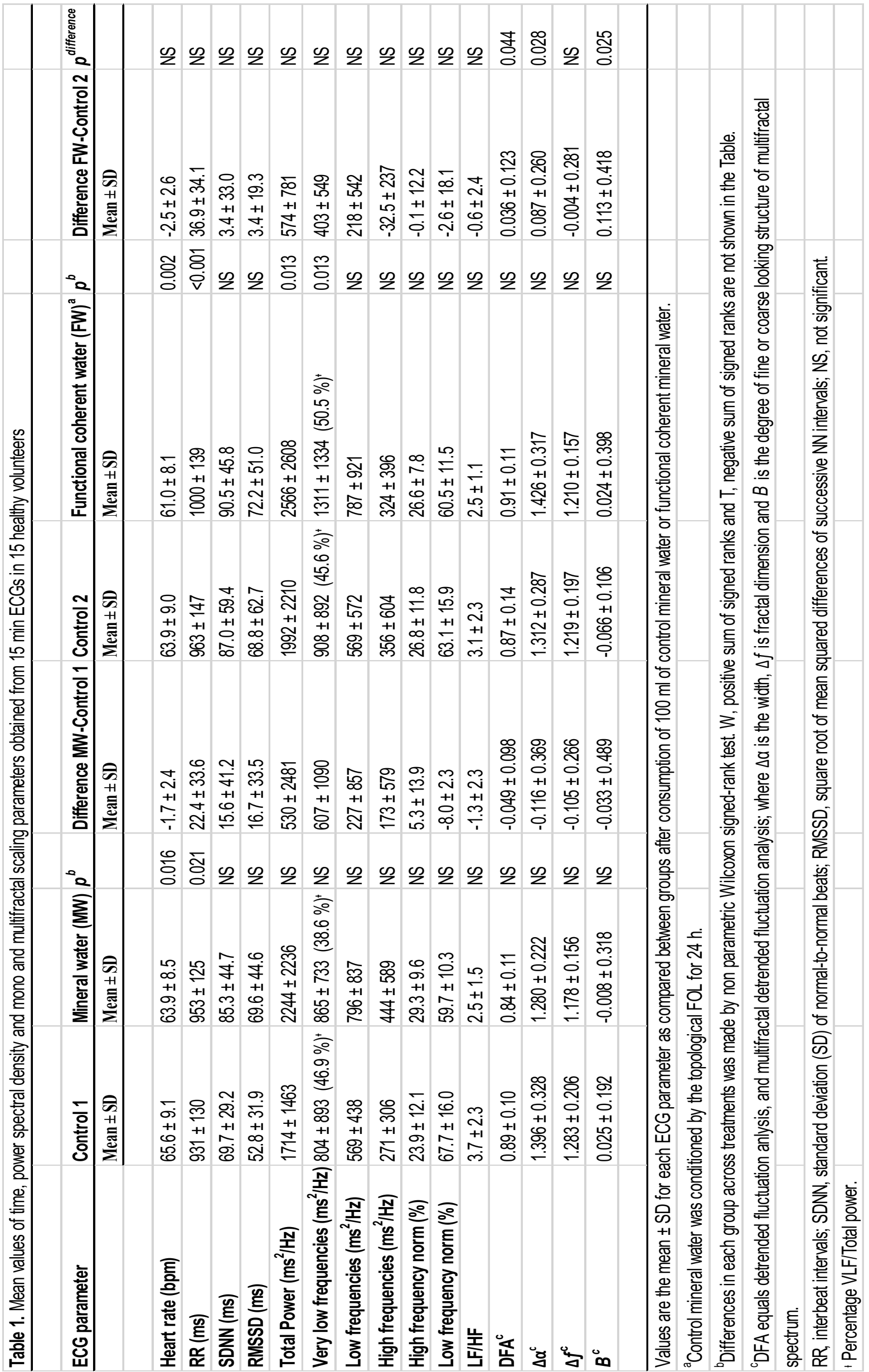




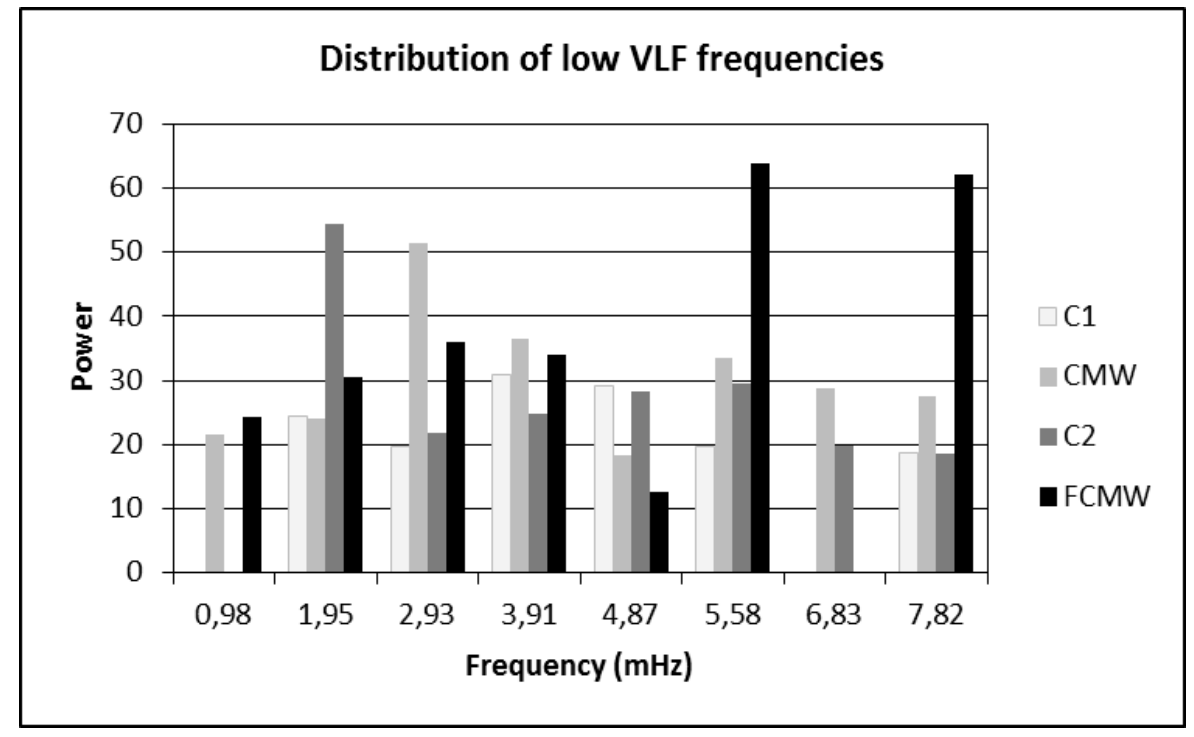

Figure 3. Distribution of low VLF signals obtained from Fourier analysis of VLF frequency spectrum $(0,98-7,82 \mathrm{mHz})$ (Figure 2). The main frequency peaks represent VLF oscillations in control experiments $1(\mathrm{C} 1)$ and $2(\mathrm{C} 2)$, and after ingestion of control mineral water (CMW) or functional coherent mineral water (FCMW).

The larger the $\Delta \alpha$ value, the richer the signal in the structure. A larger $B$ value (positive) for functional coherent mineral water indicated a left-skewed multifractal spectrum shape and a relative dominance of lower fractal exponents corresponding to a more smooth-looking structure. The lower $B$ value (negative) for control mineral water indicated a rougher structure of a less multifractal scaling spectrum. No correlation between BMI and time or frequency domain and mono or multifractal scaling parameters could be identified, indicating that the effect of control mineral and functional coherent mineral water were independent of the variation in body mass index (BMI) (data not shown).

Drinking control mineral water revealed that the mean plasma concentration of salivary IgA was significantly lower $(p=0.018)$ opposed to the unaffected plasma concentration after ingestion of functional coherent mineral water (Figure 4).

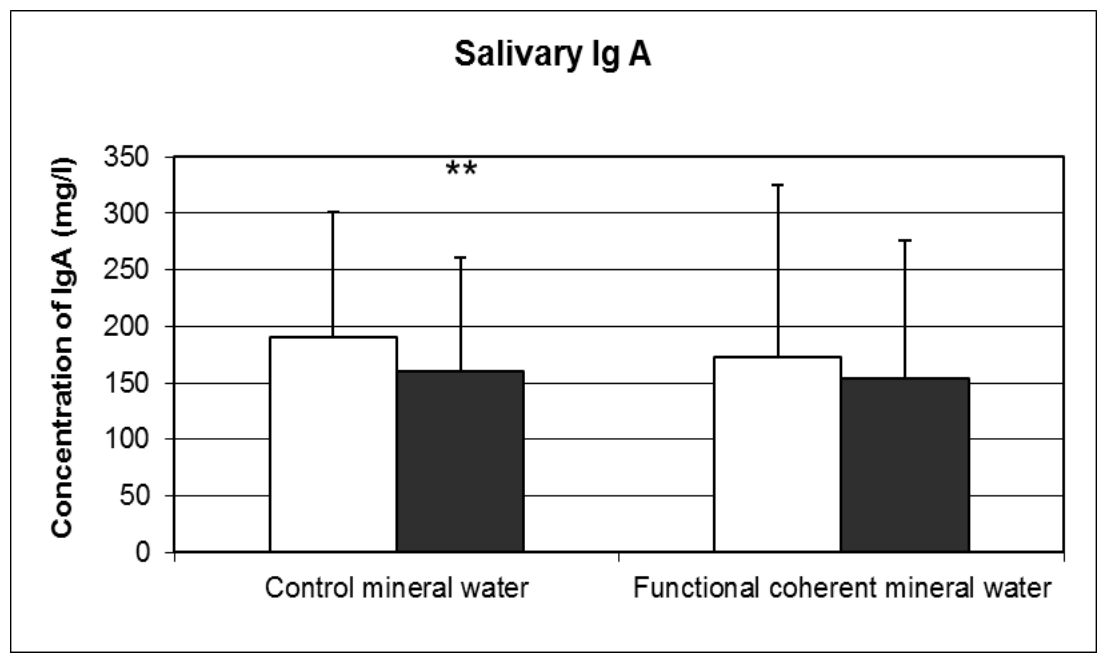

Figure 4. Unstimulated salivary concentration of immunoglobulin A ( $\operatorname{IgA})$ in Functional coherent mineral water in relation to control mineral water values $(* * p=0.018)$. White bars represent results before experiment and the black bars represent results in subjects after drinking control or functional coherent mineral water. 


\section{DISCUSSION}

This study assessed the effect of drinking functional coherent mineral water on cardiovascular time and frequency domain, and mono and multifractal parameters with reflexive response on blood pressure and mucosal humoral immunity. The primary finding, besides an expected bradycardial regulative response in healthy subjects, was a significantly larger total power of the heart with concomitant increasing effect on 2-3 minute oscillations of the VLF power band, indicating alteration in the heart's intrinsic cardiac rhythm that is considered fundamental to health and wellbeing [32]. The intrinsic regulation in VLFinduced HRV was strongly coordinated to mono and multifractal scaling of heart rate dynamics, which defines self-regulative non-local adaptability with higher capacity to respond to unpredictable stimuli and stresses, a characteristic of a healthy autonomic function [2]. The result of the current study adds new information to the results of our previous findings. Firstly, the beneficial effect of intake of functional mineral water on a rapid parasympathetic restorative response, stimulated mucosal humoral immunity and a proposed self-regulative stabilization of blood pressure [46]. Secondly, the humidifying effect of indoor greenery irrigated with vortex-water, which improved a vagus-induced sympathovagal balance on the heart in healthy volunteers exposed to ordinary EMFs in visual display terminal (VDT) work [57].

The small but significant change in blood pressure in healthy subjects 20-25 minutes post-drinking was unexpected, due to the small volume of ingested water. Previous studies in young and mixed aged humans $[46,77]$, contrary to older [78] healthy humans, have shown no effects on blood pressure after ingestion of water volumes up to $500 \mathrm{ml}$. Normally, when a pressor effect is observed in e.g. patients with autonomic failure, the response was evident within five minutes after drinking started, reached a maximum after 20 to 35 minutes, and was sustained for more than 60 minutes [78]. In our previous study after intake of functional mineral water [46], the distinct difference in curve shape between control mineral water with a non-significant increase in systolic and diastolic blood pressure between 10-20 minutes post-drinking accompanied a higher mean level different from baseline between 25 to 60 minutes. The straight horizontal line from the start of drinking to 60 minutes post-drinking suggested a stabilizing effect on normal blood pressure. Accordingly, in the present study, drinking functional coherent mineral water resulted in a higher consistent mean difference in curve shape from the baseline, indicating a steady arterial systolic and diastolic blood pressure. The impact of fractal scaling along with distinct classes of non-linear interactions reveals that fractal self-regulation appears to underlie important aspects of cardiac VLFinfluence of endogeneous blood pressure rhythms on the heart, which might have considerable predictive value for health outcomes [79, 80].

The defined change in HRV implied the VLF rhythm is a specified intrinsic sympathetic oscillation modulated by nonlocal and nonlinear fractal signal processing [2], which is also involved with thermal, metabolic rate, hormonal, and visceral regulation [61], in addition to cardiac-related activity [81]. Since the VLF frequency band is sensitive to ruminative or worrisome thoughts frequently observed in many psychophysiological disorders, the signal has been considered a potentially powerful tool for autonomic regulation. When physiological activity was reflected to the patient in a self-management program, VLF rhythms served as an indicator of the patient's attempt to achieve a calm and peaceful internal state [61]. In the presence of an EMF, the influence of higher VLF signals indicated a distortion in ordinary long-term heart cycles of an extended biological duration, possibly 
affecting and inducing a long-term hormonal response to external stressors-thereby optimizing individual coping ability and the perception of control [26]. It has been proposed that the long-term cycles of the VLF frequency band originates from self-oscillations in the vasomotor part of the baroreflex loop of the heart and in heart transplant recipients without autonomic innervation. The heart has very little activity in the VLF region, indicating that the majority of the activity in this band is due to autonomic influence [26, 82].

In our previous study [57] with gaseous vortex water transpired from indoor greenery improving a vagus-induced sympathovagal balance on the heart at an external EMF load, it was concluded that the EMF contributed to the increase in VLF signals. At exposure to natural plant and water stimuli, a reduction in VLF power indicated that the natural cues actually affected involuntary responses to EMF-induced distortion in PSD regulation, and only active plants induced qualities in air conditions equal to those of the control experiment before the EMF load was applied. Additionally, the present discussion proposes that active water could be a tentative mediator of a long-range coherent consistent force acting at a distance of approximately $1 \mathrm{~m}$ between test subject and active plant. The temporary shielding effect on the distant force observed with the presence of the control plant, notwithstanding the fact that a consistent coherent space around the experimental set-up was regained when the control plant was removed, indicates that non-local coherent oscillations were amplified by the coherent oscillations of active water, strengthening the consistency and influence of non-local coherent oscillations on the VLF rhythms of the heart.

The influence from the background non-local low VLF could be related to the fact that in order to load energy in the water CDs, a resonating magnetic field is needed [42]. In higher organisms, such as humans, these fields can be induced by the nervous system. In elementary organisms, such as bacteria or yeast cells and physiological liquids, i.e. water, environmental fields, like geomagnetic fields can serve as the inducer [42, 81, 84]. These modes act as stationary fields produced by the magnetic activity occurring in the shell whose boundaries are the surface of the earth-conductive ionosphere resonant cavity, which acts as a mirror wall for VLF and Alfvén waves [83]. Aqueous solutions of bicarbonates, superoxide radicals, and other ROS, when excited, show variations in energy emitting activity that have been found to correlate with fluctuations in the geomagnetic field [84]. Accordingly, bacterial and viral DNA sequences have been found to induce low-frequency EMFs in high aqueous dilutions [82]. The formation of condensed DNA copies was triggered by the ambient geomagnetic EMF background of VLFs related to the Schaumann resonances (SR), specifically the $7.8 \mathrm{~Hz}$ band. In humans, physiological rhythms and global collective behaviours are not only synchronized with solar and geomagnetic activity, but disruptions in these fields can also invoke adverse effects upon human health and behavior [83]. It is well established that the resonant frequencies of geomagnetic Ultra Low Frequencies (ULF) $(0.006-0.2 \mathrm{~Hz})$ overlap closely with the frequencies of the cardiovascular system $(0.002 \mathrm{~Hz}-$ $0.2 \mathrm{~Hz}$ ), while SR $(7.8-51 \mathrm{~Hz})$ directly overlap with those waves of the human brain (Theta 4-7 Hz, Alpha 8-12 Hz, Beta 12-30 Hz, and Gamma 30-100 Hz). It is expected that variations in intensity in these fields have a strong correlation with changes in HRV indices, especially with changes in the VLF rhythms of HRV [84]. Based on these observations, the outcome of experimental observations from indoor greenery [57], and the constitution of a fractal quantum dissipative state of functional coherent mineral water [51], the result of the present study implies this water state a plausible amplifier of extending VLF signals compliant with those of a ULF with an enhanced dominating impact on specifically 2-3 
minutes $(5.6-7.8 \mathrm{mHz})$ long-range VLF rhythms of the heart, and different from the local effect of control mineral water on particularly 6-9 minutes $(2.9-3.9 \mathrm{mHz})$ VLF signals, which includes a generic restrainment of the entire VLF band.

The specific induction of non-local VLF power after ingestion of functional coherent mineral water reveals the presence of long-range, power law correlations, as a part of mono and multifractal correlates operating over a wide range of time scales [2]. These scaling properties imply that the non-linear regulatory systems are operating far from equilibrium, and that the specifically induced VLF rhythms are imperative in regulation of fractal dynamics of long-term heart oscillations [32]. As a test of whether heartbeat time series exhibit fractal behavior and to elucidate their correlation properties, the DFA algorithm can be applied on long-term heart rate recordings. For a healthy condition the DFA shows a scaling behavior with exponent $\alpha$ equal to 1.0, indicative of long-range correlations extending to a huge number of heartbeats. The data on the experimental group shows a growing DFA value (Table 1) consistent with improved long-range correlations with high persistence after drinking functional coherent mineral water. In contrast, the control DFA value confirmed a loss of fractal correlation properties. The strong persistence signifies that heartbeat variations from small time intervals to larger ones are positively correlated in a power-law fashion. This implies that the long-term memory should be considered in the trend prediction of fractal heart rate dynamics as an important factor [2].

In contrast to monofractals (DFA) characterized by only one singularity exponent throughout the entire signal, multifractals require a larger, and theoretically infinite, number of indices to characterize their scaling properties [85]. The multifractal curve follows a hyperbole shape, where the width of the spectrum graph, $\Delta \alpha$, indicates a non-uniform clustering structure of the heartbeat sequences. The larger $\Delta \alpha$ (Difference FW-Control 2) after ingestion of functional coherent mineral water shows that the variation of heartbeats was larger and the multifractality was stronger (Table 1). The larger the $\Delta \alpha$, the richer the signal in its structure. No change in the fractal dimension, $\Delta f$ (Difference FW-Control 2 related to Difference MW-Control 1), between the two tests indicates that the probability of the heartbeat values lying at the lowest site was equal to those of the highest site. The larger $B$ value (positive) for functional coherent mineral water, indicates a left skewed multifractal spectrum shape and a relative dominance of lower fractal components corresponding to more smooth-looking fractal structures. The left-skewed symmetry of parameter $B$ makes fractal heart rate fluctuations attractive and offers multiple interactive combinatory coherent oscillations on small scales. The differences between the two multifractal spectrums can be explained by the fact that intake of control mineral water, may induce a heterogeneous disordered state characterized by distorted structural dynamics owing to the absence of fractal long-range ordering, while the opposite capability of the coherent conditions of the ordered mineral water state is characterized by a dynamical change from disordering towards coherence. This implies that the change and difference of heartbeat multifractal parameters induced by functional coherent water should be considered in the application and recommendation of functional coherent water in overall promotion of health [2]. The defining feature of a healthy function is its adaptability, the capacity to respond to unpredicted stimuli and stresses, where the fractal non-local correlations serve as a self-organizing mechanism for highly complex processes in the heart's intrinsic cardiac nervous system that generate fluctuations across a wide range of time scales [2, 32]. The absence of adaptive dynamics of a fractal state and long-term regulatory mechanism prevents appearance of highly ordered 
periodic behaviors, which would greatly delimit functional responsiveness [2]. The loss of fractal correlations and the appearance of a scale-free system accompany a dominant nonadaptive physiological mode that is observed in many pathologies [86, 87].

The nervous system reflexively regulates the immune system in real time in accordance with the cardiovascular response, blood pressure, respiration, and other vital functions [88]. The modulation of the immune response, a cardioprotective effect on the heart [89], and promotion of energy conservation by maintaining the heart rate lower than the intrinsic effect on heart rate [89] involves parasympathetic activation of afferent vagus nerve fibre [90]. Our previous observation supported a proposed parasympathetic mediated self-regulative stabilization of blood pressure by functional water, accompanying a protective advantage of mucosal humoral immunity by the increase in secretory IgA in saliva [46]. However, in the present study the sympathetic VLF influence on a temporally and marginally time-limited increase in blood pressure, and the consistency of a steady difference in blood pressure curve shape from baseline, with a densified stabilized secretory IgA on mucosal surfaces conforms partly with the double vagal-sympathetic response hypothesis [91]. The mechanism behind this response suggests that the sympathetic pressor effect evoked by the intake of water raised an immediate counterbalanced cardiac vagal activity, which promotes a bradycardial effect $[75,76,92,93]$. From our current observations, the bradycardial response on consumption of functional coherent mineral water was proposed as elicited from a non-local and fractal organized modulation of the 2-3 minute VLF rhythm on the heart, offering a preventive and stabilizing effect on blood pressure with an additional densified protection against mucosal pathogenic colonization. Importantly, to keep autonomic balance a healthy system is constantly and dynamically changing [2]. Therefore, an important indicator of the status of health of the regulatory systems is the capacity to respond to and adjust the relative autonomic balance, e.g. the heart rate, to the appropriate state for the context the individual is engaged in at any given moment [32]. Consequently, the dynamic bradycardial regulatory response accompanying the 2-3 minute induced VLF rhythm identified a reflexively intrinsic and non-local originated ability of the physiological self-regulatory system to maintain the relative balance between the sympathetic and parasympathetic systems independent of sympathetic or parasympathetic controls or the autonomic balance between these controls as indexed by different measures of HRV [94]. The rise in normal 2-3 minute VLF power obtained in a restful sitting position appears to indicate a self-sustained and promoting healthy function. Accordingly, the apparent shift from local longer (6-9 minute) to non-local shorter (2-3 minute) VLF rhythms strengthens a self-oscillatory global sympathetic outflow from the heart's intrinsic activity, where the VLF induced change in beat-to-beat fluctuations of the heart regulate an adaptive, minor and steady rise in blood pressure [32].

The quantum properties of the functional coherent state of mineral water [51] at ambient temperature and pressure conditions relates to the quantum electrodynamic (QED) field theory [37], as evoked by the interaction between the high entropy photons of incident ordinary daylight passing the high symmetry FOL raster space. According to the theory of dissipative systems' ability to self-regulate [16], it was implied that the exchange of energy and entropy with the environment relied on the fact that the absorption of spatially selforganized low entropy photons of incident conditioned sunlight into high grade energy with low entropy displaces the equilibrium towards a dominance of coherent water CDs. Likewise, the CDs of functional coherent mineral water were characterized by a distinctive configurative coherent aqueous state with a fractal scaling low-entropy relationship in 
radiative temperature fluctuations that maintained a self-organized structural adaptation irrespective of ambient temperature [51]. When energy from the quantum field was captured by the incident photons, they were self-trapped and turned into an excited state of coherent photons oscillating in phase with the quantum EMF [37]. At this point, the Lamb-like shift, a critical number of coherent water molecules are tuned together and are enclosed within the CDs, where a phase transition occurs [37]. The coherent oscillations of the molecules in the CDs no longer require any external supply of energy. It is stabilized and self-organized on all scale levels, which in the coherent state of mineral water was identical to a strong power law relationship in the fractal scaling boundary of thermal IR emission flickering, characterized by high persistence, and the existence of CDs that enable a reservoir of quasi-free electrons [51].

CDs are spontaneous receptors of weak non-local EMF signals [37]. Water molecules become structurally clustered, not only attracting adjacent water molecules but also other guest molecules able to resonate with the same frequency [4]. With a further increase in density, the CDs become a net exporter of energy because the self-stabilized coherent state has a lower energy $(12.06 \mathrm{eV})$, than the ionization threshold of an ordinary water state (12.60 eV) [37]. Such coherent CDs contain millions of a low entropy state of non-local clustered water molecules, and a non-vanishing probability of having an infinite number of quasi-free electrons that can be donated readily to electron-acceptor systems. The quasi-free electrons, considered as the most significant property of a quantum coherent system, form frictionless vortices with an extremely long lifespan [5], lasting for days or weeks depending on a selfregulated magnetic moment that aligns coherent intrinsic and external vortices that cannot degrade thermally. In view of the novel observation in functional coherent mineral water of a VLF mediated oscillation with non-local origin, the significant decrease in heart rate, independent of changes in respiratory sinus arrhythmia (RSA) accompanied by an approximate decline (4.5-\% related to the control 2.6-\%) in intermediary aerobic mitochondrial metabolism and in the production of biological energy (i.e. adenosine triphosphate, ATP), the CDs can be a candidate in a proposed mimic role of an enzyme functioning as a source of highly excited electrons [37]. Hypothetically, in their excited coherent phase the CDs could act as a donator of excited electrons or catalyse an excitation to a fairly low activation energy to free them to be able take part in the mitochondrial electron transfer at just the activation energy of a specific enzyme corresponding exactly with the excitation energy of the relevant coherent state of clustering CDs [37]. Interestingly, a recent study has shown that drinking $500 \mathrm{ml}$ of ordinary water enhanced metabolic rate by $30-\%$ mainly fuelled by the oxidation of lipids in men and carbohydrates in women [96]. The thermogenic effect of ordinary water was suggested as linked to sympathetic activation.

In our previous study of healthy subjects drinking functional water, the change towards a restorative cardiac vagal HRV [46], which also may involve frequencies in the $0.1 \mathrm{~Hz}$ region, referred to as cardiac coherence [8], was reflected in a healthy adaptability and an inherent self-regulatory capacity and resiliency [8-12]. This finding that consumption of liquid functional water was likely to involve a condition of autonomic resonance [33] and a net sympathovagal activity on the heart, contributed to new information that high-density coherent qualities of body water participated in the regulation of cardiovascular functioning. Since the functional quality of mineral water was generated from a three-dimensional geometrical field raster space with a path-curve form of an interlocked vortex [97, 98], in terms of optics, while uniformly passing through space the radiation undergoes 
rearrangement and transforms into a non-linear series of wave structures with fractal ordering. This low entropy state of highly ordered photons, assumed to generate coherent EMF oscillations in water in the LF frequency region around $0.1 \mathrm{~Hz}$, was proposed as acting as a non-local resonating amplifier of intrinsic cardiac coherent oscillations. A concomitant influence on the processing of coherent oscillatory information, as well as non-local functioning of liquid functional water or conditioned air from indoor greenery [57], is likely. Considering the VLF coupled multifractal cardiac properties obtained from drinking functional coherent mineral water, the high symmetry state of the path-curve form generated from a two-dimensional FOL gains encoded information of physical matter in threedimensional space [98]. A counter-acting spherical force of two vortices flowing in opposite directions reconstructs a higher state of spatial symmetry of incident photons of ordinary light at different frequencies that are organized virtually according to fractal wave dynamics in space passing the geometry. Self-trapped higher symmetry state photons captured by water CDs may oscillate in resonance with collective coherent electron vortices and molecular rhythms within the excited CDs [4]. In the ordered coherent water state, an incremental oscillatory change in a dynamic concentric temperature gradient aligns with formation of two interlocking vortices flowing in opposite directions with a slight difference in thermal IR temperature between the two gradient path-curve forms [51]. Accumulated, condensed and structurally ordered energy distributed into several concentric temperature zones of the two interlocking vortices were aligned with a broadening temperature interval towards lowered temperatures in the segments with most ordered CDs.

To get a collective performance of water CDs, which can give rise to resonance with intrinsic heart VLF rhythms, would require a uniform rate of energy loading from, e.g., a magnetic field for all the involved CDs [42]. A plausible interaction with geomagnetic ULF can affect the human cardiovascular system, because several ULFs are in a comparable range with those of the human heartbeat and its rhythms [99]. The external non-local background load of conditioned EMF photons in water CDs in resonance with, e.g., the frequency of ULF geomagnetic field pulsations that can be biotropic [100], specifically, stable continuous pulsations (Pulsations continuous; Pc). According to Class Pc4 (time period 45-150 sec) or Pc5 (time period 150-600 sec) [101], may contribute to the rise in cardiac intrinsic 2-3 minute VLF rhythms [82]. This observation adds partly new information that VLF rhythms obtained from two-dimensional path-curves represent a strengthening of healthy multifractal heart rate dynamics [2] strongly correlating with non-local self-regulation mechanisms underlying the fact that the VLF rhythm is the primary source of this rhythm within the heart itself [32]. Additionally, assessing the regulatory autonomic capacity of functional mineral water, three-dimensional path-curve forms obtained from non-local ordering of conditioned mineral water [46] or air from indoor greenery [57] appear to represent an index of vagally mediated improved HRV, which induce auto-coherent heart rhythms typically amplifying signals in the LF-region around $0.1 \mathrm{~Hz}$. Future studies will be designed on 24-hour HRV recordings to elucidate how different high symmetry two and three-dimensional path-curve forms can oscillate in resonance with quantum fluctuations or geomagnetic continuous ULF pulsations in order to be important biotropic agents of the geomagnetic background influence on cardiac regulatory ULF and VLF rhythms, strongly associated with overall health status. Moreover, the emergent impact on vagally induced HRV, particularly mid LF rhythms as an index of emotional self-regulation and restoration and maintenance of metabolic energy 
resources, will be investigated in detail, to determine the mirror resonant characteristics between high symmetry fractal path curve forms and non-local EMF frequencies.

\section{CONCLUSION}

In this study on healthy humans drinking functional coherent mineral water, a bradycardial regulative response on heart rate was accompanied by a significant increase in the 2-3 minute VLF band, reflecting a strengthening in mono and multifractal heart rate dynamics. These results imply that the VLF rhythms of the heart are in fact stimulated after consumption of a conditioned coherent water state, which entails a highly ordered and multifractal arrangement of the molecular configuration of the physiological "body water" with an apparent non-local EMF identity. Therefore, the change in energy expenditure may suggest that functional coherent mineral water stimulates a more efficient metabolism, and that the energy can partly be extracted directly from the coherent domains of ingested functional coherent mineral water. The stabilized blood pressure response and a densified protection of mucosal surfaces link with an involuntary emergent intrinsic autonomic response. The increase and change in the VLF rhythm, which is produced by the heart itself, thereby act as a regulator of mono and multifractal heart rate dynamics, which help restore autonomic regulative capacity, a fundamental and profound indicator of the status of individual's health and well-being.

List of abbreviations used: FCMW, functional coherent mineral water; CMW, control mineral water; VLF, very low frequency; HRV, heart rate variability; ECGs, electrocardiograms; HF, high frequency; LF, low-frequency; ULF, ultra low frequency (ULF); ANS, autonomic nervous system; CDs, coherent domains; EMF, electromagnetic field; ROS, reactive oxygen species; FOL, Fibonacci optical lattice; NRW, natural reductive mineral water; EW, electrolysed water; HBA1c, glycated hemoglobin A1; DNA, deoxyribonucleic acid; 8-OHdG, 8-hydroxy-2-deoxyguanosine; LDL, low density lipoprotein; GOT, glutamic oxaloacetic acid transaminase; $\gamma$-GTP, $\gamma$-glutamyl transpeptidase; PSD, power density parameters; IgA, Immunoglobulin A; BMI, Body mass index; ECG, Electrocardiography; mean HRT, mean heart rate; mean NN, normal to normal heartbeats; 5$\operatorname{minSDNN}=$ SDNN, standard deviation of the normal to normal heartbeats; RMSSD, the square root of the mean squared differences of successive N-N intervals; LF/HF, absolute LF to absolute HF power; DFA, Detrended fluctuation analysis; VDT, visual display terminal; VBA, Visual Basic for Applications; PET, polyethylene terephthalate; QED, quantum electrodynamics; RSA, respiratory sinus arrhythmia; ATP, adenosine triphosphate

Competing interest: The authors declare they have no competing interests.

Authors' contributions: BJ conceived and coordinated the study, analyzed the data and drafted the manuscript. SS performed the statistical analysis. Both authors read and approved the final manuscript.

Acknowledgements and Funding: The authors are grateful to the volunteers who participated in this study and to Johan Kronholm for development and adjustment of the multifractal software. This study was partly supported by Ekhagastiftelsen, Stockholm, Sweden. The founding source had no role in any part of the study. 


\section{REFERENCES}

1. Cannon WB: The James-Lange theory of emotion: a critical examination and an alternative theory. Am J Physol. 1987, 100(3-4): 567-586.

2. Goldberger GL, Amaral LAN, Hausdorff JM, Ivanov PCh, Peng CK, Stanley HE: Fractal dynamics in physiology: Alterations with disease and aging. PNAS. 2002, 99(19): 2466-2472.

3. Del Giudice, Pulselli RM, Tiezzi E: Thermodynamics of irreversible processes and quantum field theory: an interplay for the understanding of ecosystem dynamics. Ecol Model. 2009, 220: 1874-1879.

4. Brizhik L, Del Giudice E, Jörgensen SE, Marchettini N, Tiezzi E: The role of electromagnetic potentials in the evolutionary dynamics of ecosystems. Eco Model. 2009, 220: 1865-1869.

5. Marchettini N, Del Giudice E, Voeikov V, Tiezzi E: Water: A medium where dissipative structures are produced by coherent dynamics. J Theor Biol. 2010, 265 : 511-516.

6. Shirahata S, Hamasaki T, Teruya K: Advanced research on the health benefit of reduced water. Trends in Food Sci \& Technol. 2012, 23: 124-131.

7. McCraty R, Atkinson M, Tiller W, Rein G, Watkins AD: The effects of emotions on short-term power spectrum analysis of heart-rate variability. Am J Cardiol. 1995, 76: 1089-1093.

8. McCraty R, Atkinson M, Tomasion D, Bradley RT: The coherent heart: heart-brain interactions, psychophysiological coherence, and emergence of system-wide order. Boulder Creek, California: Institute of Heartmath; 2009.

9. McCraty R, Childre D: Coherence: bridging personal, social, and global heath. Altern Ther Health Med. 2010, 16(4): 1024.

10. Singer DH: High heart rate variability, marker of healthy longevity. Am J Cardiol. 2010, 106(6): 910.

11. Geisler FC, Kubiak T, Siewert K, Weber H: Cardiac vagal tone is associated with social engagement and self-regulation. Biol Psychol. 2013, 93(2): 279-286.

12. McCraty R, Zayas MA: Cardiac coherence, self-regulation, autonomic stability, and psychosocial well-being. Front Psychol. 2014, 5: 1090.

13. Shaffer F, McCraty R Zerr CL: A healthy heart is not a metronome: an integrative review of the heart's anatomy and heart rate variability. Front Psychol. 2014, 5: 1040.

14. Vitiello G: Current states, fractals and brain waves. New Math Natural Comp. 2009, 5: 245-264.

15. Vitello G: Fractals and the Fock-Bargmann representation of coherent states. Third international representation of coherent states (QI-2009). Lexture notes in artificial intelligence edited by Goebel et al. In Quantum interaction. Edited by Bruza P. Berlin, Heidelberg: Springer -Verlag: 2009, 6-16.

16. Prigogine I, Nicolis G: Selforganisation in non-equilibrium systems, from dissipative structures to order through fluctuations. New York: Wiley; 1977, 90-160.

17. Del Giudice E: Formation of dissipative structures in liquid water. Water. 2009, 2: (Suppl.1):14-15.

18. Heart rate variability standards of measurements, physiological interpretation, and clinical use. Task Force of the European Society of Cardiology and the North American Society of Pacing and Electrophysiology. Circulation. 1996, 93(5): 10431065. 
19. Brigger JT Jr, Fleiss JL, Steinman RC, Rolnitzky LM, Kleiger RE, Rottman JN: Frequency domain measures of heart period variability and mortality after myocardial infarction. Circulation. 1992, 85(I): 164-171.

20. Shah AJ, Lampert R, Goldberg J, Veledar E, Bremner JD, Vaccarino D: Posttraumatic stress disorder and impaired autonomic modulation in male twins. Biol Psychiarty. 2013, 73(II): 1103-1110.

21. Lampert R, Bremner JD, Su S, Miller A, Lee F, Cheeema F, Goldberg J, Vaccarino V: Decreased heart rate variability is associated with higher levels of inflammation in middle-age men. Am Heart J. 2008, 156(4); 759-759.

22. Carney RM, Freedland KE, Stein PK, Miller GE, Steinmeyer B, Rich MW, Duntley SP: Heart rate variability and markers of inflammation and coagulation in depressed patients with coronary heart disease. J Psychosom Res. 2007, 62(4): 463-467.

23. Theorell T, Liljeholm-Johanson Y, Björk H, Ericson M: Saliva testosterone and heart rate variability in the professional symphony orchestra after "public faintings" of and orchestra member. Psychoneuroendrocrinology 2007, 32(6): 660-668.

24. Kleiger RE, Stein PK, Bigger JT Jr: Heart rate variability:measurement and clinical utility. Ann Noninvas Electrocardiol. 2005, 10(1): 88-101.

25. Axelrod S, Gordon D, Ubel FA, Shannon DC, Berger AC, Cohen RJ: Power spectrum analysis of heart rate fluctuation: a quantitative prob of beat-to-beat cardiovascular control. Science. 1981, 213(4504): 220-222.

26. Cerutti S, Bianchi AM, Mainardi LT: Special analysis of heat rate variability signal. In Heart rate variability. Edited by Malik M, Camm AJ. New York: Armonk Futura Publishing Company, Inc. 1995, 63-74.

27. Murphy DA, Thompson GW, Ardell JL, MaCraty R, Stevenson RS, Sangalang VE, Cardinal R, Wikinson M, Craig S, Smith FM, Kingma JG, Amour JA: The heart reinnervates after transplantation. Ann Thorac Surg. 2000, 69(6): 1769-1781.

28. Ramaekers D, Ector H, Vanhaecke J, van Cleemput J, van de Werf F: Heart rate variability after cardiac transplantation in humans. Pacing Clin Electrophysiol. 1996, 19(12 Pt I): 2112-2119.

29. Amour JA: Neurocardiology-anatomical and functional principles. Boulder Creeek, California: Institute of HeartMath. 2003.

30. Kember GC, Fenton GA, Amour JA, Kalyaniwalla N: Competition model for aperiodic stochastic resonance in a Fitzhugh-Nagumo model of cardiac sensory neurons. Phys Rev E Stat Nonlin Soft Matter Phys. 2001, 63 4Pt I): 041911.

31. Kember GC, Fenton GA, Collier K, Amour JA: Aperiodic stochastic resonance in a hysteretic population of cardiac neurons. Physical Rev. 2000, 61(2): 1816-1824.

32. McCraty R, Shaffer F: Heart rate variability: New perspectives on physiological mechanisms, assessments of self-regulatory capacity, and health risk. Global Adv Health Med. 2015, 4(1): 46-61.

33. Tiller W, McCraty R, Atkinson M: Cardic Coherence: A new non-invasive measure of autonomic nervous system order. Altern Therap. 1996, 2: 52-65.

34. Wernet PH, Nordlund D, Bergmann U, Cavalleri M, Odelius M, Ogasawara H, Näslund LA, Hirsch TK, Ojamäe L, Glatzel P, Pettersson LG, Nilsson A: The structure of the first coordination shell in liquid water. Science. 2004, 304: 995-998.

35. Huang C, Wikfeldt KT, Tukoshima T, Nordlund D, Harada Y, Bergmann U, Niebuhr M, Weiss TM, Horikawa Y, Leetmaa M, Ljungberg MP, Takahashi O, Lenz A, Ojamäe L, Lyubartsev AP, Shin S, Pettersson LG, Nilsson A: The inhomogeneous structure of water at ambient conditions. PNAS. 2009, 106: 15214-15218. 
36. Arani R, Bono I, Del Giudice E, Preparata G: QED coherence and the thermodynamics of water. Int J Modern Phys. 1995, B9: 1813-1841.

37. Del Giudice E, De Ninno A, Fleischmann M, Mengoli G, Milani M, Talpo G, Vitiello G: Coherent quantum electrodynamics in living matter. Electromagn Biol Med. 2005, 24: 199-219.

38. Hirokawa N, Tanaka Y, Okada Y, Takeda S: Nodal flow and the generation of leftright asymmetry. Cell. 2006, 125: 33-45.

39. Hove JR, Koster RV, Forouhar AS,Acevedo-Bolton G, Fraser SE, Gharib M: Intracardic fluid forces are an essential epigenic factor for embryonic cardiogenesis. Nature, 2003, 421: 172-177.

40. Shirahata S: Reduced water for preventing diseases. In Animal cell technology. Basic $\&$ applied aspects. Edited by Shirihata S. Dordrecht: Kluwer Academic Publishers; 2002, 12: 25-30.

41. Voeikov VL: In Fundamental role of water in bioenergetics. Edited by Belussov LV, Voeikov VL, Martynuk VS. New York: Springer; 2007, 89-104.

42. Montagnier L, Del Giudice E, Aissa J, Lavallee C, Motschwiller S, Capolupo A, Polcari a, Romano P, Tedeschi A, Vitiello: Transduction of DNA information through water and ele AT and ctromagnetic waves. Electromagn Biol Med. 2015, 34(2): 106112

43. Brizhik LS, Del Giudice E, Tedeschi A, Voeikov VL: The role of water in the information exchange between the compartments of an ecosystem. Eco Model. 2011, 222: $2869-2877$.

44. Scientific concepts of functional foods in Europe: Consensus document. Br J Nutr. 1999, 81(1): S1-S27.

45. Asp N-G: Health effects of probiotic and preprobiotic foods. Scand J Nutr. 2001, 45: 57.

46. Johansson B: Effects of functional water on heart rate, heart rate variability, and salivary immunoglobulin A in healthy humans: A pilot study. J Alter Compl Med. 2009, 15(8): 871-877.

47. Shirahata S, Li Y, Hamasaki T, Gadek Z, Teruya K, Kabayama S, Otsubo K, Morisawa S, Ishii Y, Katakura Y: Redox regulation by reduced waters as active hydrogen doners and intracellular ROS scavengers for prevention of type 2 diabetes. In Cell technology for cell products. Edited by Smith R. Dordrecht: Springer; 2007, 99-101.

48. Sugihara S, Suzuki C, Hatanaka K: The mechanisms of activation of substances by minimal catalyst water and application in keeping foods fresh. Water 2001, 3: 87-94.

49. Ikeda S, Takata T, Komoda M, Hara M, Kondo JN, Domen K, Tanaka A, Hosono H, Kawazoe H: Mechano-catalysis a novel method for over-all water splitting. Phys Chem Chem Phys. 1999, 1: 4485-4491.

50. Domrachev A, Roldigin GA, Selivanovsky DA: Rule of sound and liquid water a dynamically unstable polymeric system in mechano-chemically activated processes of oxygen production on earth. J Phys Chem. 1992, 66: 851-855.

51. Johansson B, Sukhotskya S: Allometric scaling behaviour - A quantum dissipative state implies a reduction in thermal infrared emission and fractal ordering in distilled coherent water. Water. 2012, 3: 100-121.

52. Hayashi H, Kawamura M: Clinical application of electrolyzed-reduced water. In Animal cell technology: Basic \& applied aspects. Edited by Shirahata S. Dordrecht: Kluwer Academic Publishers; 2002, 12: 31-36. 
53. Gadek Z, Li Y, Shirihata S. Influence of natural reduced water on relevant test parameters and reactive oxygen species concentration blood of 320 diabetes patients in the prospective observation procedure. In Animal cell technology; Basic \& applied aspects. Editied by Lijima S, Nishijima KI. Dordrecht: Springer; 2006, 14: 377-385.

54. Osada K, Li YP, Hamasaki T, Abe M, Nakamichi N, Teruya K: Anti-diabetes effects of Hita Tenryosui water, a natural reduced water. In Animal cell technology: Basic \& applied aspects. Edited by Ikura K. Dordrecht: Springer; 2010, 15: 307-313.

55. Matsubayashi N, Hisamoto T, Murao N, Hara T: About effect of so called reduced water on diabetes patients. In The abstract book of the $46^{\text {th }}$ Kyushu Regional Meeting of Japan Diabetes Society. 2008, 42.

56. Higashikawa F, Kuriya T, Noda M, Sugiyama M. Verification of improving action of mineral water on lipid metabolism in clinical trials. In Abstract book of the $7^{\text {th }}$ meeting of the Japanese Society of Preventive Medicine. 2009, 20.

57. Johansson B: Heart rate and heart rate variability response to the transpiration of vortex-water by Begonia Eliator plants to the air in an office during visual display terminal work. J Alter Compl Med. 2008, (8): 993-1003.

58. Ulrich RS, Simons RF, Losito BD: Stress recovery during exposure to natural and urban environments. J Environ Psychol. 1991, 11: 201-230.

59. Ulrich RS, Lundén O, Eltinge JL: Effects of exposure to nature and abstract pictures on patients recovering from heart surgery. Science. 1984, 224: 420-421.

60. Laumann K, Gärling T, Stormark KT: Selective attention and heart rate responses to natural and urban environments. Environ Psychol. 2003, 23: 125-134.

61. Gevirtz R: Resonant frequency training to restore autonomic homeostasis for treatment of psychological disorders. Biofeedback. 2000, 27: 7-9.

62. Henry JP, Stephens PM: Stress, health and the social environment: A sociobiologic approach to medicine. New York, Heidelberg, Berlin: Springer Verlag; 1977.

63. Goldberger AL: Software for calculation of DFA was downloaded from [www.physionet.org/physiotools/dfa(2009)]. 2009.

64. Ihlen EFA: Introduction to multifractal detrended fluctuation analysis in matlab. Front Physiol 2012, 3: 141.doi:10.3389/fphys.2012.00141

65. Kantelhardt JW, Zschiegner SA, Koscielny-Bunde E, Havlin S, Bunde A, Stanley HE: Multifractal dtrended fluctuation analysis of nonstationary time series. Physica A. 2002, 316: 87-114.

66. Huang Z-W, Liu C-Q, Zhang B: Monofractal and multifractal scaling analysis of $\mathrm{pH}$ time series from Dongting lake inlet and outlet. Fractals 2010, 18: 309-317.

67. Kronholm J: The multifractal DFA was performed using a VBA script for Microsoft Excel developed at Kronholm Development AB, Sweden. 2015 [www.kronholmdevelopment.se] Algorithms and software were based on Kantelhardt (65), Huang (66) and Ihlen (64), as well as VBA scripts by Martin Sewell [www.longmemory.com]

68. Sigview spectrum analyser. Software for calculation of FFT based signal analysis was downloaded from [www.sigview.com]. 2014.

69. McCraty R, Atkinson M, Rein G, Watkins AD: Music enhances the effect of positive emotional states on salivary IgA. Stress Med. 1996, 12: 167-175.

70. Shirtcliff EA, Granger DA, Schwartz E, Curran MJ: Use of salivary biomarkers in biobehavioral research: Cotton based sample collection methods can interfere with salivary immunoassay results. Psychoneuroimmunology. 2001, 26: 165-173. 
71. Reiber H, Peter JB: Cerebrospinal fluid analysis: Disease-related data patterns and evaluation programs. J Neurol Sci 2001, 184: 101-122.

72. Johansson B. Synchronized water and production thereof. (Patent application, 000119). February 13. 2008.

73. Wiggins PM: Enzymes and two states of water. J Biol Phys Chem. 2002, 2: 25-37.

74. Watterson JG: The pressure pixel. Unit of life. BioSystems. 1997, 41: 141-152.

75. Graziano G: On the size dependence of hydrophobic hydration. J Chem Soc. 1998, 94: 3345-3352.

76. Steel EA, Merz Km, Selinger A, Castleman AW: Mass-spectral and computational free-energy studies of alkali-metal ion-containg water clusters. J Phys Chem. 1995, 99: 7829-7836.

77. Routledge HC, Chowdhary S, Coote JH, Townend JN: Cardiac vagal response to water ingestion in normal human subjects. Clin Sci. 2002, 103; 157-162.

78. Jordan J, Shannon JR, Black BK, Ali Y, Farley M, Costa F, Diedrich, Robertson R, Biaggioni I, Robertson D: The pressor response to water drinking in humans: A sympathetic reflex. Circulation. 2000, 101: 504-509.

79. Raab F, Schaffer E, Cornèlissen G, Hallberg F: More on the dentist's role in prevention of high blood pressure. Chronobiologia. 1993, 20: 245-250.

80. Cornèlissen G, Hallberg F, Otsuka K, Singh B Chen C-H: Chronobiology predicts actual and proxy outcomes when dipping fails. Hypertension. 2007, 49: 237-239.

81. Axelrod S: Components of heart rate variability. Basic studies. In Heart rate variability. Edited by Malik M and Camm AJ. NewYork Armonk: Futura Publishing Company, Inc; 1995, 3-19.

82. Bernadi L, Valle F, Coco M, Calciati A, Sleight P: Physical activity influences heart rate variability and very-low-frequency components in Holter electrocardiograms. Cardiovasc Res. 1996, 32(2): 234-237.

83. McCraty R, Deyhle A, Childre D: The global coherence initiative: Creating a coherent planetary standing wave. Glob Adv Health Med. 2012, 1(1): 64-76.

84. Voeikov VL, Vilenskaya ND, Minh Ha D, Malyshenko SI, Buravleva EV, Yablonskaya OI, Timofeev KN. The stable state of bicarbonate aqueous systems. Rus J Phys Chem A. 2012, 86(9): 1407-1415.

85. Pobachenko SV, Kolesnik AG, Borodin AS, Kalyuzhin VV: The contingency of parameters of human encephalograms and schaumann resonance electromagnetic fields revealed in monitoring studies. Complex Syst Biophys. 2006, 51(3): 480-483.

86. Stanley HE, Amaral LAN, Goldberger AL, Havlin S, Ivanov P Ch, Peng CK: Physica A. Statistical physics and physiology: Monofractal and multifractal approaches. 1999, 270: 309-324.

87. Ivanov PCh, Amaral LAN, Goldberger AL, Havlin S, Rosenblum MG, Struzik Z, Stanley HI: Multifractality in human heartbeat dynamics. Nature. 1999, 399: 461-465.

88. Amaral LAN, Ivanov PCh, Aoyagi N, Hidaka I, Tomono S, Goldberger AL, Stanley EH, Yamamoto Y: Behavioral-independent features of complex heartbeat dynamics. Phys Rev Lett 2001, 86: 6026-6029.

89. Tracey KJ: The inflammatory reflex. Nature. 2002, 420: 853-859.

90. Buch AN, Coote JH, Townend JN: Mortality, cardiac vagal control and physical training-what's the link? Experiment Physiol. 2002, 87(4): 234-235.

91. Borovikova LV, Ivanova S, Zang M, Yang H, Botchkina GI, Watkins LR, Wang H, Abumrad N, Eaton JW, Tracey KJ: Vagus nerve stimulation attenuates the systematic inflammatory response to endotoxin. Nature. 2000, 405: 458-462. 
92. Pecanha T, Paula-Riberiro M, Campana-Rezende E, Bartels R, Marins JC, de Lima JR: Water intake accelerates parasympathetic reactivation after high-intensity exercise. Int J Sport Nutr Exerc Metabol. 2014, 24: 489-496.

93. Brown CM, Barberini L, Dulloo AG, Montani JP: Cardiovascular responses to water drinking: does osmolality play role? Am J Physiol Regul Integr Comp Physiol. 2005, 289(6): R1687-1692. Doi:00205.2005.

94. Berntson GG, Norman GI, Hawkley LC, Cacioppo JT: Cardiac autonomic balance versus cardiac regulatory capacity. Psychophysiol. 2008, 45(4): 643-652.

95. Del Giudice E, Tedeschi A: Water and autocatalysis in living matter. Electromag Biol Med. 2009, 28: 46-52.

96. Boschman M, Steiniger J, Hille U, Tank J, Adams F, Sharma AM, Klaus S, Luft S, Jordan J: Water-induced thermogenesis. J Clin Endocrinol Metabol. 2003, 88: 6015-6019.

97. Edwards L: The vortex of life. Edinburgh: Floris Books; 1993.

98. Chown M: Our world may be a giant hologram. New Scientist. 2009, 2691: 24-27.

99. Kleimenova NG, Kozyreva OV, Breus TK, Raoport SI: Pc1 geomagnetic pulsations as a potential hazard of the myocardial infarction. J Atmosphere Solar-Terrest Phys. 2007, 69(14): 1759-1764.x

100. Samsonov SN, Kleimenova NG, Kozyreva OV, Petrova PG: The effect of space weather on human heart diseases in subauroral latitudes. Atmospheric Ocean Phys. 2014, 59(7): 719-7127.

101. Jacobs JA: Geomagnetism. Dyfed: Academic Press; 1989. 